\title{
Review and Alignment of Tag Ontologies for Semantically-Linked Data in Collaborative Tagging Spaces
}

\author{
Hak Lae Kim ${ }^{\diamond}$, Alexandre Passant ${ }^{\triangleright}$, John G. Breslin ${ }^{\diamond}$, Simon Scerri ${ }^{\diamond}$ and Stefan Decker ${ }^{\diamond}$ \\ ${ }^{\diamond}$ Digital Enterprise Research Institute, National University of Ireland, Galway, IDA Business Park, Newcastle Road \\ Galway, Ireland \\ Email: \{haklae.kim, john.breslin, simon.scerri, stefan.decker\}@ deri.org \\ $\triangle$ LaLIC, Université Paris-Sorbonne, 28 rue Serpente, 75006 Paris, France \\ Email: alexandre.passant@paris4.sorbonne.fr
}

\begin{abstract}
As the number of Web 2.0 sites offering tagging facilities for the users' voluntary content annotation increases, so do the efforts to analyze social phenomena resulting from generated tagging and folksonomies. Most of these efforts provide different views for the understanding of various web activities. Results from various experimental research should be utilized to improve existing approaches underlying tagging data and contribute further to weaving the Web. However, in practice, there are not enough solutions taking advantage of these results. Even though we can mine social relations via tagging data, it proves no worth for users if this data cannot be reused.

In this paper we propose a solution for tag data representation which allows data reuse across different tagging systems. To achieve this goal, we analyze current social tagging practices, existing folksonomy usage as well as Semantic Web approaches to data annotation and tagging. We survey and compare existing tag ontologies in an attempt to investigate mapping possibilities between different conceptual models. Finally, we present our method for federation among existing ontologies in order to generate re-usable, semantically-linked data that will underly tagging data.
\end{abstract}

\section{INTRODUCTION}

The practice of tagging online data became popular thanks to Web 2.0 services such as Flickr ${ }^{1}$ and del.icio.us ${ }^{2}$. While Web 2.0 principles and tools like blogs and wikis offer new principles for end-users to publish content, tagging provides them with a simple way to control how they index their data. By annotating content with free keywords, users (whether creators or consumers) decide which metadata will be associated with their content, without any need to resort to existing, pre-defined and authoritative indexing schemes. While tags are simple keywords, they can be used for various levels of metadata. Indeed, even though they are mainly used as descriptive metadata (eg. tags about the content of a blog post), they can also be used as structural metadata (eg. "w3c" tag to identify a document from w3 website) or administrative (e.g. "gpl" or "creative-commons" to identify licence issues)

${ }^{1}$ http://www.flickr.com

${ }^{2} \mathrm{http}: / /$ del.icio.us about the tagged object. Moreover, tagging can be used for various purposes, and Golder and Huberman [1] identified seven different functions that tags can play for end users, from opinion to self-reference, while Marlow et al. [2] argue than tagging can also reflect a social view about users.

Nevertheless, while tagging is attractive from a user point of view when annotating data, since it does not require much effort to be applied, it raises various issues when retrieving content. Davis [3] thus argues that the advantages of using tags when producing data are lost when retrieving it. We believe that some of those issues can be solved by adding semantics to tags, the tagging process and related to objects like tag clouds. Indeed, formal models to define these concepts are needed to leverage tagging via the Semantic Web and to automate certain tasks. Moreover, a common set of semantics will allow people to share and exchange tags between applications. For example, such a semantic modelling process could enable the answering of queries like "What are the most popular tags that Bob uses on Flickr that identify places and that are also used by Alice on del.icio.us". Some work regarding links between tagging and Semantic Web technologies has already been done, and will be covered in this article.

The paper is structured as follows. We first provide an overview of existing folksonomies and the issues they raise in an information retrieval process. Next we will provide an overview of work that defines semantics for tags and tagging. A number of tag ontologies, each focusing on particular aspects of tagging, have been designed. After presenting the goal of each ontology we show how we propose to align these different models and how each can be used in a complete tagging. We outline which ontologies are used in each part of the tagging process via a practical use case. Here we mainly place on models providing a common framework and guidelines for using the tag ontologies, rather than implementable features like automation based on the unified model. We finally conclude with an outline for future work in the area, which is at the frontier of a combined Semantic Web and Web 2.0, 
also known as the Social Semantic Web [4].

\section{TAg Ontologies}

A lot of scholarly work has been done on the topics of folksonomies and the Semantic Web, but relatively few studies have been carried out on tagging representation at a semantic level. However, a formal representation for tagging plays important roles to reflect various experimental results on the Web. Many studies have been performed in a lot of disciplines via innovative approaches. However, without consistent structures and semantics, contributions of these studies are unable to analyze the social phenomenon relating the folksonomies. In order to operate social ecosystems on the Web, we need various technical and social analyses for folksonomies as well as formal representation for adopting the results. The semantics of tagging data is primarily about an agreement on the meaning among people or a community in the social space. A common semantics provides a way to share tag representation among services. We now provide an overview of a number of existing efforts that had the common aim of representing the concepts and operations of tags and tagging.

\section{A. Gruber's Conceptual Model}

Gruber [5] describes tagging as a relation between:

- An object, i.e. the resource to be tagged. For example, a bookmark, a picture, a blog post etc.

- A tag, i.e. the tag associated with the resource

- A tagger, i.e. the agent - more generally a person - that created the link between the tag and the object

- A source, i.e. the space where the tagging action has been performed, e.g.: Flickr, del.icio.us

Notably, Gruber defines the source as the scope of namespaces or universe of quantification for objects. This allows one to differentiate between tagging data from different systems and is the basis for collaborative tagging across multiple applications. Mika [6] already represented the tagging action from a theoretical point of view, but did not use this notion of source that Gruber introduces. Yet, while this model is widely commended, there is no implementation.

\section{B. Tag Ontology}

Using the tripartite Tagging (User, Resource, Tag) model Newman et al. [7] defined an ontology of tags and tagging, simply called the Tag Ontology, that describes the relationship between an agent, an arbitrary resource, and one or more tags. Thus, in his ontology, the three core concepts Taggers, Tagging, and Tags are used to represent the tagging activity. Contrary to Gruber [5], it does not represent the source of the tagging action. Yet, this ontology has been implemented (in OWL), is available on the Web, and is currently used in some projects such as Revyu.com ${ }^{3}$, a review website combining Web 2.0 and Semantic Web technologies.

Notably, in this ontology tags are represented as instances of the tags: Tag class which is assigned custom labels, i.e. the

\footnotetext{
${ }^{3}$ http://www.revyu.com
}

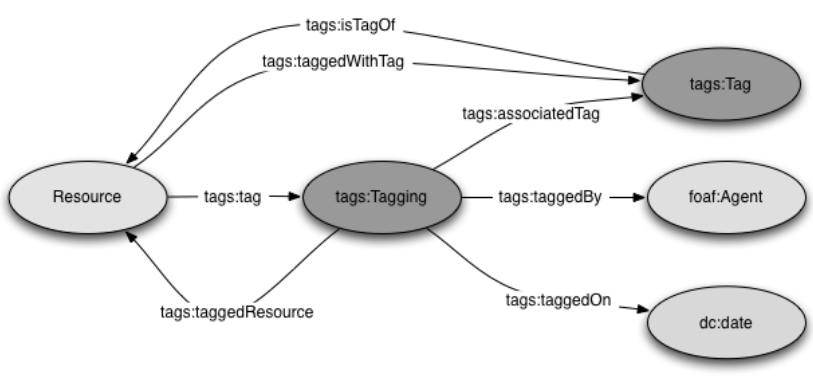

Fig. 1. Tagging activity using Tag Ontology

string representing the tag as seen by the user. Being instances of a class means that they are assigned a URI. URIs are a key feature of the Semantic Web, since, contrary to simple literals, they can be used as subject of triples, while literals can be only used as objects. This way, tags - identified by URIs can be linked together and people can semantically represent connection and similarities between tags. For this purpose the ontology introduces a tags: related property. Yet, this relation does not have much semantics, since it does not define the nature of the relation, e.g. if this a linguistic variation or because it identifies a similar topic. Another limitation is that the ontology does not define any cardinality constraint on the number of labels a Tag can have. This can raise problems since it allows a Tag instance to have two completely disjoint labels (i.e. a Tag instance with labels "RDF" and "Paris"), which makes no sense from a tagging point of view.

Still, this ontology reuses pre-defined Semantic Web vocabularies, making it compliant with existing standards. SKOS properties are used to model relations between tags and the Tag class itself inherits from skos: Concept. DublinCore is used to represent the date of a tagging action, with subproperties of $d c$ : date. Finally, the ontology relies on FOAF to identify the tagger of a tagging action thanks to foaf:Person.

\section{SCOT}

The Social Semantic Cloud of $\mathrm{Tags}^{4}$ ontology aims to describe the structure and the semantics of tagging data and to offer social interoperability of the data among heterogeneous sources[8].

Both Tagcloud and Tag class in SCOT play a role to be able to represent social and semantic context of tagging, since both classes include users, tags, and resources and additional information to clarify tags' semantics. scot: TagCloud has properties that describe a certain user, tag spaces, number of tags, posts and co-occurrences and their frequencies, as well as updated information. The property scot: contains links scot:TagCloud to a set of scot:Tag instances. scot:Tag, as a subclass of tags:Tag from the Tag Ontology, describes a tag that is aggregated from individual tagging activities. This class has several properties such as

\footnotetext{
${ }^{4}$ http://scot-project.org
} 


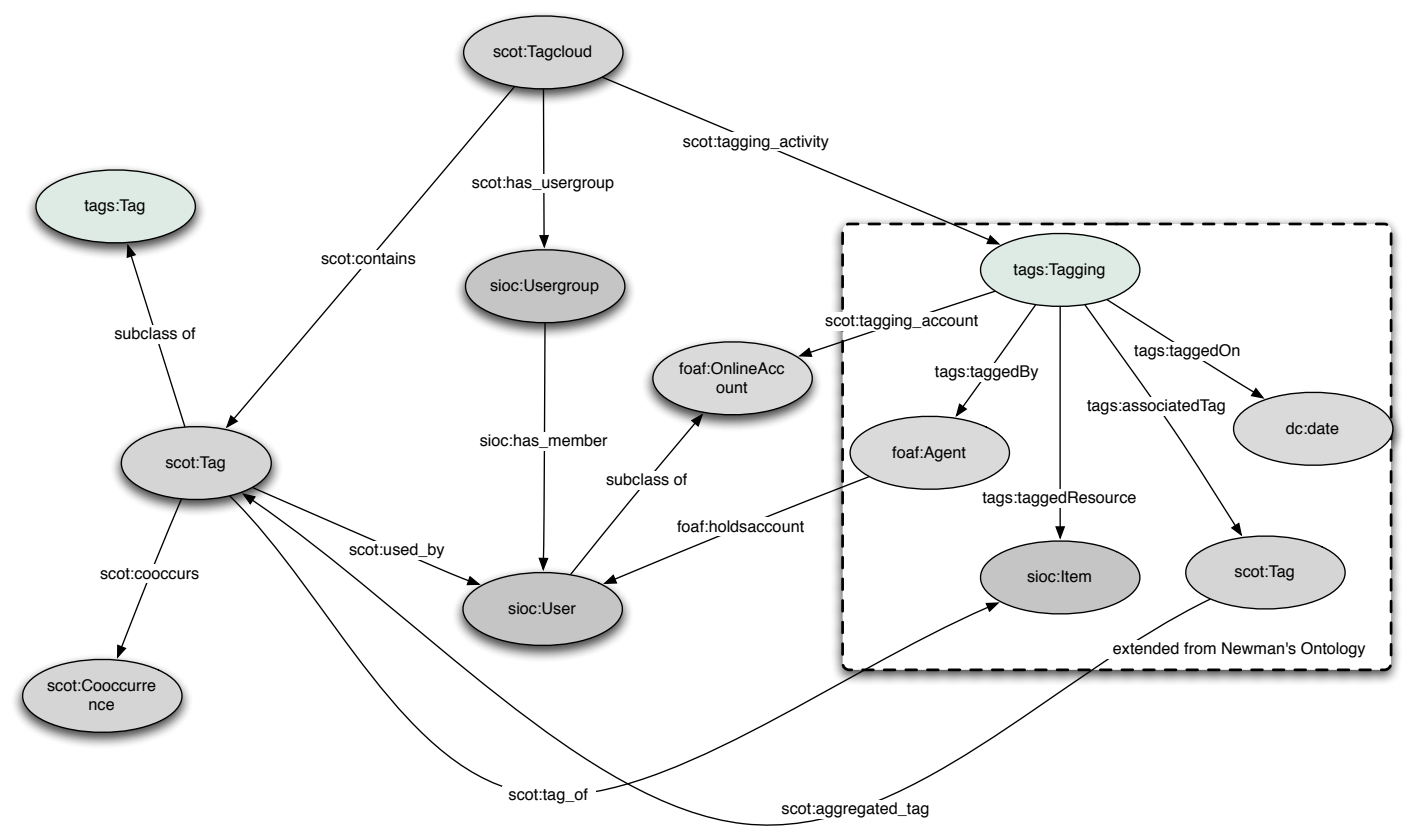

Fig. 2. Simplified folksonomy model in SCOT

scot:spelling_variant, scot: synonym to solve tag ambiguities. We called these properties "linguistic property" since they focus on representing the meaning of the relationship between tags from a linguistic point of vie. In addition, this class has also properties to describe occurrence of a tag (i.e. scot:frequency). A tag itself has its own frequency. The frequency is not unique, but it is an important feature to distinguish or compare with other tags. We called it a "numerical property" The properties have their own numerical values by computing.

It is important to note that SCOT uses concepts and properties of Newman's model. As shown in Figure 1, the Tagging class represents tags themselves, the resources that are being tagged, and the users that create these tags (tags:taggedBy). The scot:Tagcloud class connects tags:Tagging instances via the property scot:tagging_activity. In SCOT, we try to define the range values of tagging properties more specifically. For instance, tags:taggedResource has sioc:Item as a range value whereas tags:associatedTag has scot:Tag as its range. Individual tags in tags:Tagging are mapped to a resource with scot:Tag instance and then these tags are represented by a collection of tags underlying a scot:Tagcloud. Moreover, the property scot:tagging_account represents an account of users in online services. Figure 2 illustrates the SCOT ontology model with integrating Newman's model.

At an individual level, created tags are ad-hoc/informal and must be relevant to a tagger and the tagger's view. Thus, tag sharing has to support aggregated views while keeping the local context of each tagging activity. SCOT provides a tagging social structure for seamless tag sharing across heterogeneous users, applications or sources. For instance, suppose that a user uses the tag ' $w e b$ ' three times in three different instances of tags:Tagging. The instance for the tag gather each tag with the URI, the property scot:aggregated_tag has URIs for the each tag and the property scot:tag_of is linked to sioc:Item. Moreover, this class represents not only a tag label, but also its absolute and normalized occurrences, spelling variants, and hierarchical structures (i.e. skos:broader and skos: narrower) with SKOS. Listing 1 shows a snippet of SCOT data.

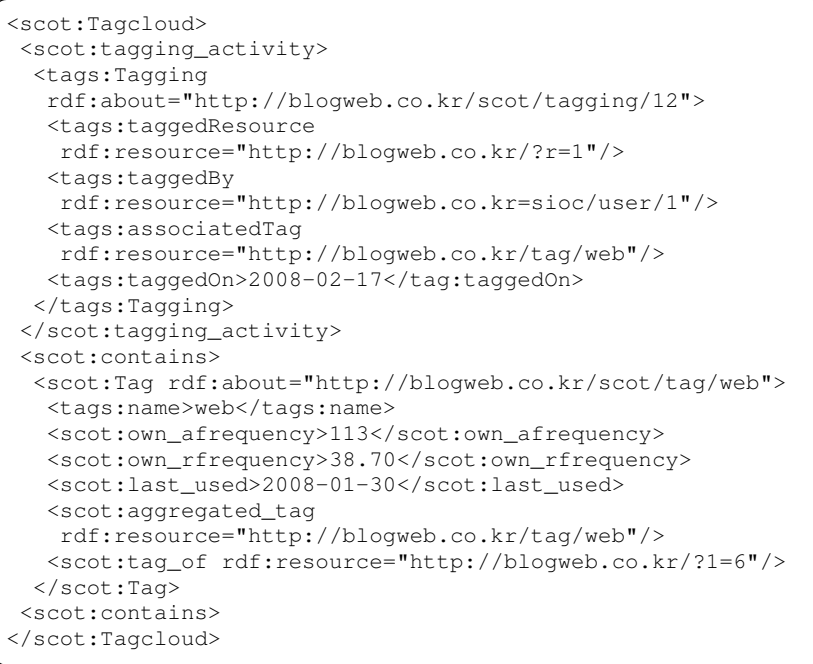

Listing 1. Example of SCOT data in RDF/XML 


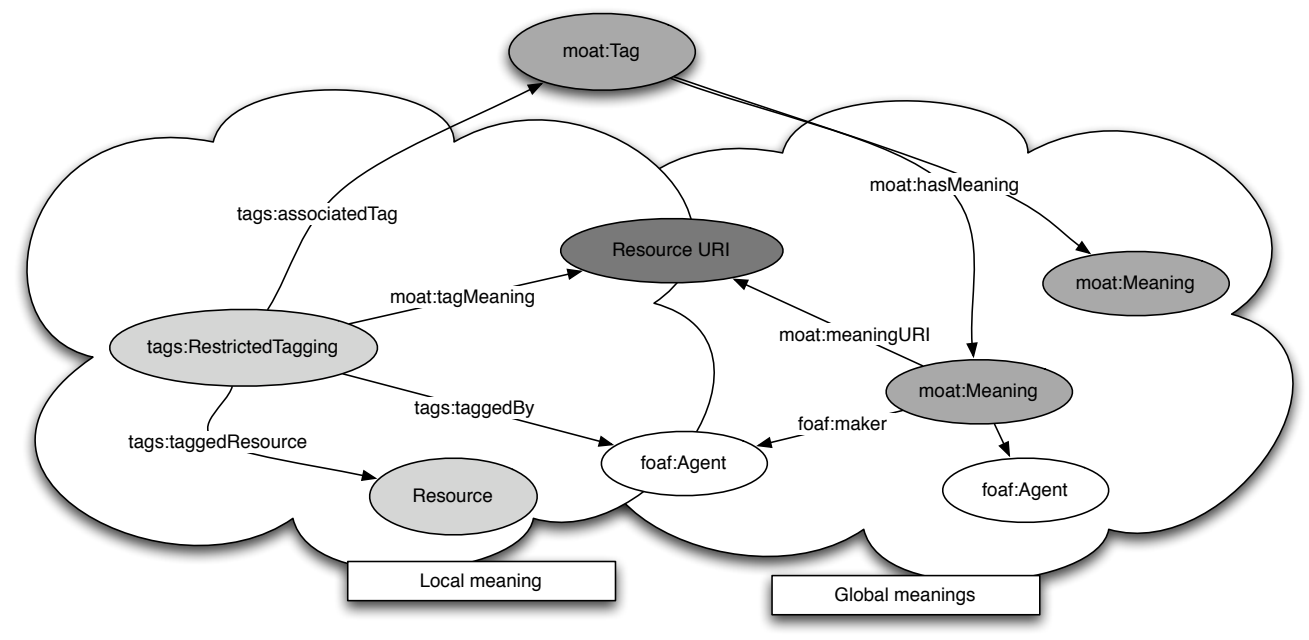

Fig. 3. Tags' local and global meaning in MOAT

SCOT allows the exchange of semantic tag metadata for reuse in social applications and enables interoperation amongst data sources, services, or agents in a tag space. These features are a cornerstone to being able to identify, formalize, and interoperate a common conceptualization of tagging activity at a semantic level. SCOT Exporter for WordPress ${ }^{5}$ can extract tagging data from WordPress and then generate SCOT instance in the blog. This process is totally automatically performed with real time updates. So far, the SCOT ontology has been used in the int.ere.st web site which provides search, bookmarking and integrating tagging data among heterogenous users, sources, or applications [8].

\section{MOAT}

MOAT's $^{6}$ - Meaning Of A Tag - goal is to provide a Semantic Web model to define the meaning of tags in a machine-readable way [9]. To achieve it, MOAT defines:

- the global meanings of a tag, i.e. the list of all meanings than can be related to a tag in a complete folksonomy;

- the local meaningof a tag, i.e. the meaning of a tag in a particular tagging action.

Indeed, for instance, the tag "paris" can mean - depending on the user, the context and other factors - a city in France, a city in the USA, or even a person. Yet when someone uses it in a tagging action, it has a particular meaning, for example the french capital. Thus, MOAT extends the usual tripartite model of tagging action to the following quadripartite model: Tagging(User, Resource,Tag, Meaning). Using MOAT, those meanings (both global and local) can be defined without ambiguity by the tagger. MOAT provides a machine-readable format, using a particular ontology, to allow computers to understand these meanings, relying on

\footnotetext{
${ }^{5} \mathrm{http}: / /$ scot-project.org/applications/wp-exporter/

${ }^{6} \mathrm{http}: / /$ moat-project.org
}

URIs of existing concepts from knowledge bases as DBpedia ${ }^{7}$, GeoNames $^{8}$, or even corporate knowledge bases to define it.

Figure 3 shows how MOAT models those meanings and reuses the Tag Ontology. MOAT introduces a Tag class as a subclass of Newman's Tag one. This subclass addresses one of the problem of the Tagging Ontology we referred to earlier, and through an OWL cardinality constraint it is only allowed to have one unique label for a given Tag instance.

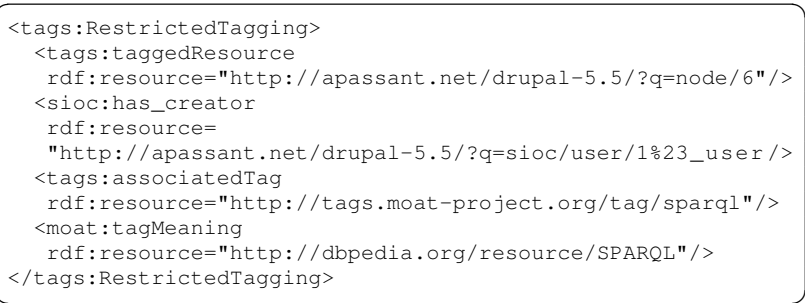

Listing 2. Example of MOAT tagging action

Each tag is linked to one or more moat:Meaning instances, which represent the meaning(s) of a tag without any context. Each meaning must have one unique URI identifying it (eg: ihttp://dbpedia.org/resource/Paris;), and be linked to the agents that defined this meaning, relying on FOAF. In that way, in the folksonomy space, meanings of tags are related to the URIs of people who assigned it: Meanings $($ Tag $)=\{($ Meaning,$\{U s e r\})\}$. To represent the context of a tag in a certain tagging action, using the quadripartite model defined before, MOAT relies on the tags: RestrictedTagging class from Newman's ontology, and introduces a moat:tagMeaning property that allows to link to the meaning of the tag in this particular context, as the previous snippet of code shows (Listing 1)

\footnotetext{
${ }^{7}$ http://dbpedia.org

${ }^{8} \mathrm{http}: / /$ geonames.org
} 
Moreover, MOAT introduces a social aspect that lets people share their tags - and their meanings - within a community by subscribing to a MOAT server, as they could do with the Annotea annotation server. They subscribe to a tag server in which they can share and update tag meanings, and use it when tagging content. When a user tag content, the client queries the server to retrieve tag meanings and let the user choose which one is the most relevant one, regarging the context. While it provides a model to represent those meanings, there is no automation, or Natural Language Processing to assist the user in chosing a new or existing tag when querying a post. Yet, the MOAT client for Drupal ${ }^{9}$ features interaction with the Sindice $^{10}$ search engine to help users chose a new URI if no relevant meaning is found. MOAT will also, in the future, rely on social networking to give higher priority to tags used by friends within a community when suggesting meanings for a given tag.

Thanks to this framework and its model, MOAT aims to provide an easy way to bridge the gap between free-tagging and semantic indexing. While users can still benefit from the simplicity of free-tagging when annotating content, linking to URIs offers a way to solve tagging ambiguity (a single tag can be related to different URIs) and heterogeneity (various tags can be related to a single URI). Moreover, using MOAT, tagged content can be linked to URIs of reference datasets, leveraging tags and tagged content to the Linked Data web ${ }^{11}$. Then, relationships defined in those datasets can be used to suggest relevant content, e.g. suggesting posts tagged "paris" from posts tagged "france" since related concepts are interlinked in DBpedia, solving the usual problem of lack of organisation in tag systems.

\section{E. Other Related Work}

A study regarding a formal specification for folksonomies has been carried out on various applications. Some efforts ${ }^{12}$ 13 discussed representing tagging data in an early stage, although no specific representation methods resulted. Knerr [10] describes the concept of tagging in the "Tagging Ontology". The ontology consists of time, user, domain, visibility, tag, resource, and type. Although his ontology covers different aspects (e.g. visibility), main ideas and concepts largely overlap with those in the Tag Ontology. The NEPOMUK Annotation Ontology $(\mathrm{NAO})^{14}$ provides vocabulary that is commonly required to annotate resources on the Social Semantic Desktop ${ }^{15}$ vision, which defines a user's personal information environment as a source and end-point of the Semantic Web. In SIOC, the Tag class is given as the object of sioc:topic to indicate the keywords assigned to a Post or Item [11].

Table I summarizes core concepts and characteristics of the mentioned ontologies. Gruber's conceptual model represents

\footnotetext{
${ }^{9}$ http://moat-project.org/clients

${ }^{10} \mathrm{http}: / /$ sindice.com

${ }^{11} \mathrm{http}: / /$ linkeddata.org

${ }^{12} \mathrm{http}: / /$ tagschema.com/blogs/tagschema/

${ }^{13} \mathrm{http} / / / \mathrm{blogs}$. sun.com/bblfish/entry/search_tagging_and_wikis

${ }^{14} \mathrm{http}: / /$ www.semanticdesktop.org/ontologies/nao/

${ }^{15} \mathrm{http} / / /$ nepomuk.semanticdesktop.org/
}

a tagging activity of individual users consisting of a relation with five parameters, i.e. the basic tagging model plus source and polarity. In particular, Newman's Tag Ontology aims to model of the tripartite representation of tagging and a temporal aspect. This ontology can be considered a basic model for representing tagging data, and in fact the ontology has been extended by other models. Meanwhile, core concepts of SCOT and MOAT do not match in Table I because they have different goals. The former describes tagclouds sharing tags among a set of users and applications, while the latter extends the tripartite tagging relation with a meaning representation that can be shared between users and used in tagging actions.

There is a number of projects related to tagging. The TagCommons project ${ }^{16}$ focuses on sharing and interoperating over tagging data. The Tagora project ${ }^{17}$ aims to provide the "Semiotic Dynamics of online Social Systems" through data analysis, modeling and theoretical constructions of social tagging. The OpenTagging project ${ }^{18}$ also strives to make tagging data open, more universal, and apply it across any number of social tagging sites. These projects would benefit if there is a means of adopting and using tag ontologies, thus bridging Semantic Web and Web 2.0 technologies, even if they themselves had no Semantic Web vocabulary underlying tagging data.

Some tools also rely on Semantic Web technologies to offer advanced tagging features to their users. Gnizr is a personal bookmark manager that allow people to link their tags together, using the SKOS [12] vocabulary, and to tag and retrieve content using these same tags. SweetWiki [13] is a semantic wiki in which users can organize the tags they use to annotate their pages. Moreover, this approach reuses the wiki principles, since the tag categorisation is not restricted to a given user, but is open to anyone using the wiki.

Finally, on a smaller extent, microformats rely on a rel-tag ${ }^{19}$ microformat that can be used to mark-up keywords on HTML documents and define them as tags. These can then be extracted by search engines as Technorati does. Yet, as all microformats, their semantics are less powerful in comparison to what can be done with the previously mentioned RDF models, and with RDF in general.

\section{Federating tAG ONTOLOGIES}

As we highlighted in the previous section, each ontology was designed for a specific use case. A single ontology does not satisfy some tagging process and queries to support all full-fledged aspects in terms of collaborative tagging. Thus, there is a need to align those ontologies, so that using one ontology at a certain point will not allow using another one, for the same tag or tagging action on a next level. This would enable sets of tags to interlink, thus maximizing the usefulness of tagging data. It is worth noting that the tagging process cannot be separated from the resources to which a set of tags

\footnotetext{
${ }^{16} \mathrm{http}: / /$ tagcommons.org

${ }^{17} \mathrm{http} / / /$ www.tagora-project.eu

${ }^{18} \mathrm{http}: / /$ opentagging.org

${ }^{19} \mathrm{http}: / /$ microformats.org/wiki/rel-tag
} 
TABLE I

COMPARISON OF TAG ONTOLOGIES.

\begin{tabular}{|c|c|c|c|c|c|c|c|c|c|}
\hline \multirow{2}{*}{ Ontology } & \multirow{2}{*}{ Namespace } & \multicolumn{5}{|c|}{ Core Concepts } & \multirow{2}{*}{ Format } & \multirow{2}{*}{ Update } & \multirow{2}{*}{ Applications } \\
\hline & & Who & What & When & Where & How & & & \\
\hline Gruber & - & Tagger & Tag & TagAssertTime & TagSource & Tagging & - & - & - \\
\hline Tag Ontology & $\operatorname{tags}^{a}$ & foaf:Agent & Tag & taggedOn & taggedResource & Tagging & OWL & Dec. 2005 & revyu.com \\
\hline TagOnt & - & Tagger & Tag & isTaggedOn & hasTagged & Tagging & OWL & Jan. 2007 & - \\
\hline Echarte et al. & - & User & Tag & hasDateTime & Source & - & OWL & 2007 & - \\
\hline SCOT & $\operatorname{scot}^{b}$ & sioc:User & Tag & updated & tagspace & $\begin{array}{c}\text { tags: } \\
\text { Tagging }\end{array}$ & OWL & $\begin{array}{c}\text { March } \\
2008\end{array}$ & $\begin{array}{l}\text { int.ere.st } \\
\text { relaxseo.com } \\
\text { www.openlinksw.com }\end{array}$ \\
\hline NAO & nao $^{d}$ & Party & Tag & $\begin{array}{c}\text { created } \\
\text { modified }\end{array}$ & Resource & - & NRL & $\begin{array}{c}\text { August } \\
2007\end{array}$ & NEPOMUK \\
\hline
\end{tabular}

${ }^{a}$ http://www.holygoat.co.uk/owl/redwood/0.1/tags/

${ }^{b}$ http://scot-project.org/scot/ns

${ }^{c}$ http://moat-project.org/ns\#

${ }^{d}$ http://www.semanticdesktop.org/ontologies/nao/ontologies/2007/08/15/nrl\#

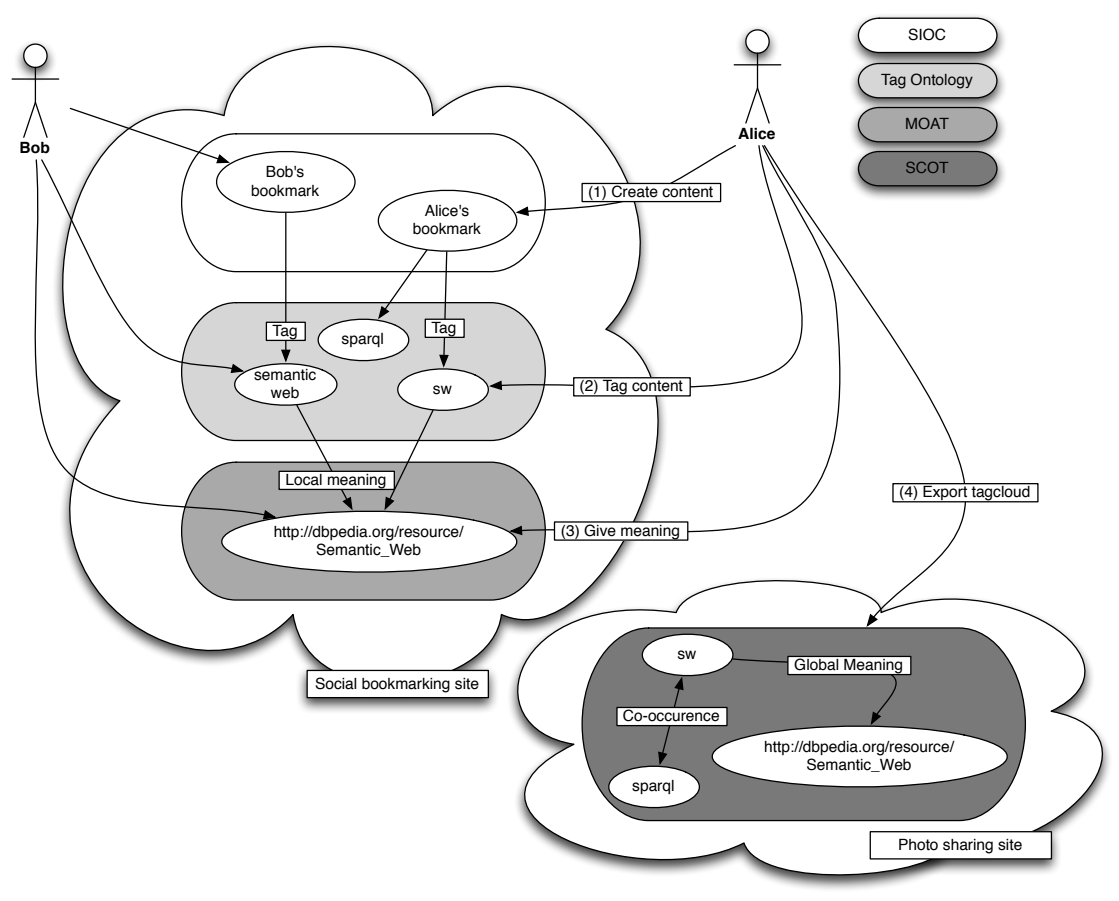

Fig. 4. Use case for defining the meaning of tags and for linking resources

is assigned and neither to the users who performed the tagging. In order to make it possible for this federation to support the tagging process, we should consider to use more extensive vocabularies such as SIOC, FOAF, SKOS, or DC.

Aligning RDF vocabularies has the advantages of providing an open, standardized access mechanism to enable people to share their data on the Web. The starting point for federating ontologies is in the "four tenets of Linked Data" as mentioned by Berners-Lee in an article [14] and coined in [15]. Our work can pave the way for making social links in tagging data via RDF vocabularies and of constructing more concrete practical cases.

Part of our work thus focused on how to federate these ontologies, and defining a use case for each stage of the process thanks to this federation. In particular, we will consider the federation among SCOT, MOAT and SIOC, since the ontologies have been used and updated in communities.

\section{A. Prerequisites}

As we explained before, the Tag Ontology from Newman provides a nice basis for describing tagging ontologies. In fact, SCOT and MOAT are currently using it to represent a Tag. Yet, 


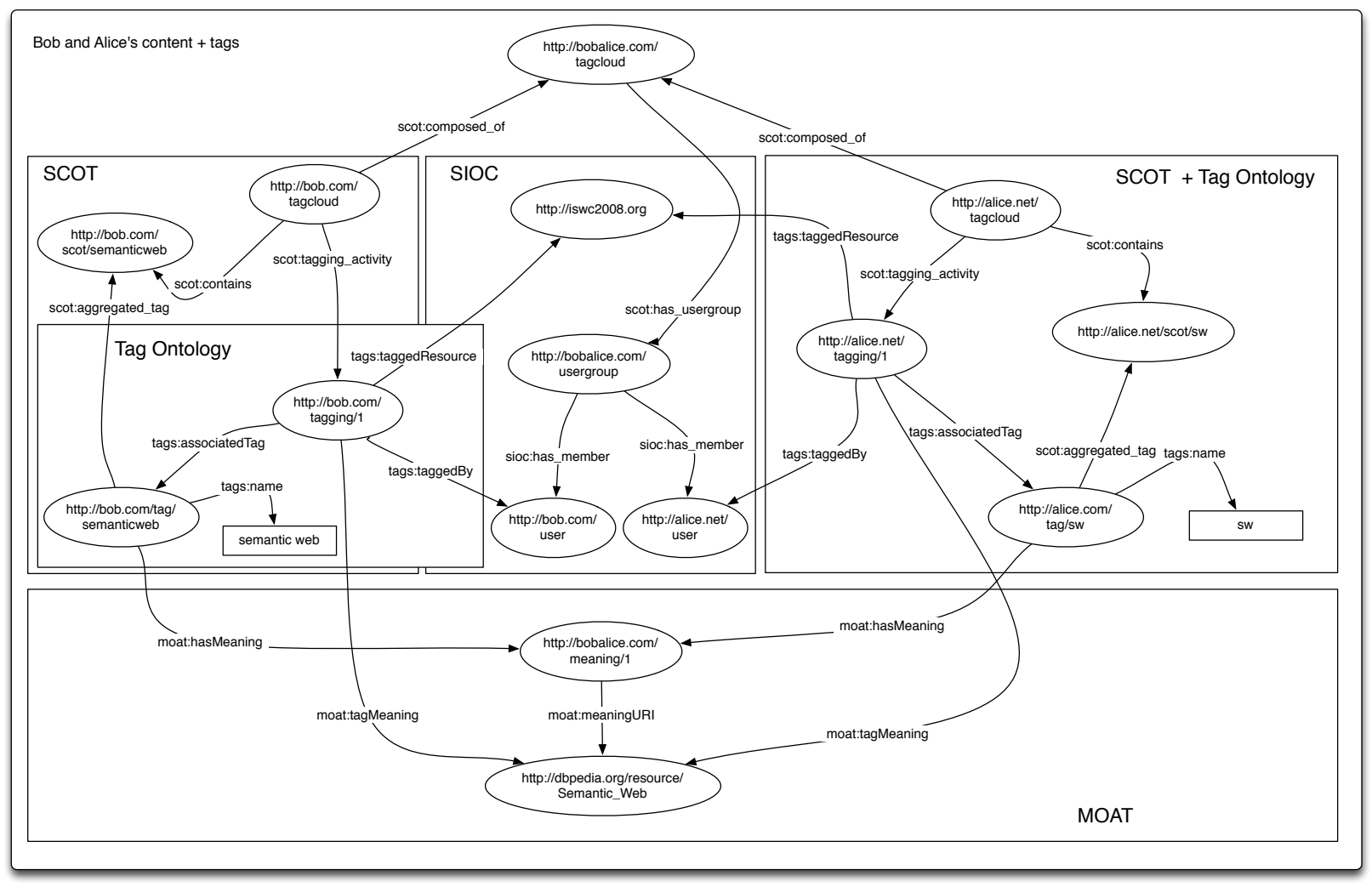

Fig. 5. Federation among RDF vocabularies

SCOT currently does not define cardinality restriction for its label, whereas MOAT does. Having such a restriction in SCOT would be helpful, since it deals with synonyms as well as casesensitive variations of a tag. Having a single unique label for each tag would thus make sense. In order to align the Tag representation of all these ontologies, having the cardinality restriction in SCOT and subclassing the MOAT tag from SCOT Tag would make sense. Moreover, in order to align MOAT and SCOT one step further, we suggest that SCOT uses the RestrictedTagging to represent tagging actions, rather than the single Tagging object. In this way each SCOT tagging action could be easily extended to MOAT, and each MOAT Tagging action could then be exported into SCOT and its tagclouds representation.

\section{B. Defining meaning of tags}

In order to represent how these ontologies, once aligned, could be used together, Fig. 4 represents the following use case, and shows which ontologies are used at each stage.

- First, Alice and Bob both add a website about a Semantic Web conference on their social bookmarking service. The bookmark is modeled thanks to SIOC and its type module (1).

- Then, both of them tag it, with different tags, 'sw' for Alice, and 'semantic web' for Bob (2).
- Since this service is MOAT compliant, they both add a meaning to their tag, which, in spite of different tags, is the same URI, i.e. $<$ http://dbpedia.org/resource/Semantic_Web> (3).

- Finally, Alice decides to export her tagcloud to her photo manager to add pictures of the conference, using SCOT. Since the photo manager is also a consumer for the same MOAT server as the social bookmarking tool, the tags of the tagcloud keep their global meaning from one service to another, so that she can reuse the tag 'sw' to bookmark her pictures, and give it the same meaning. This would also allow Bob's friend to use a new tag 'semantic_web' and re-use the same meaning URI from DBpedia. (4)

This scenario shows how tagging can be 'translated' from one site to another, while keeping all its properties thanks to the combination of Tag Ontology, SCOT and MOAT; in order to annotate any SIOC-described content.

\section{Sharing Tag Clouds}

Let's look from a different angle at the same situation. If Alice and Bob use SIOC on the sites, all posts could be represented by SIOC instances and they can define a meaning of a certain tag underlying MOAT. It might be a solution because such RDF vocabularies provide a method to interlink different 
sources on a semantic level. In addition, it might be possible to generate a social networks between two users by analyzing the meaning of tags. Let's assume that Alice and Bob add a meaningful URL '<http://dbpedia.org/resource/Semantic_Web >' in order to define the meaning of the tag ' $s w$ ' on their posts.

After producing the metadata through the publishing and tagging stage, Alice and Bob might be able to semantically link each other's underlying tag meaning. This meaning would be shared between their bookmark and tag (Fig. 5). However, this bookmark and tag are just one instance where items tagged by Alice and Bob can share the same meaning. It is thus useful to expose each individual user's tagging data semantically. These semantics, in the form of MOAT meanings, can then be shared between users with a social connection, supported by SIOC. Fig. 5 illustrates how Alice and Bob can share their resources including users, resources, tags, and the meaning of tags.

People will be able to create an object, assign a tag to the object, give a meaning to this tag in that tagging context, and then export their tag cloud to another service using various ontologies that link to each other. This process follows the previous process to represent resources in SIOC and to define a meaning of tags in MOAT.

Although we do not yet implement a system to realise the proposed model, we plan to integrate SCOT and MOAT exporters into the SparqlPress ${ }^{20}$ add-on for WordPress, which provides producers and consumers of Social Semantic Web data for this popular blogging platform.

\section{CONCLUSION}

Social tagging has become essential for Web 2.0 and Semantic Web applications. As its popularity increases, so do research efforts concerning the pros and cons of this social phenomenon. We believed that the results of these efforts can be studied and combined to improve existing approaches to tagging. In this paper, we surveyed and reviewed a number of tag ontologies whose aim is to represent tagging data semantically. After comparing the characteristics of each of these ontologies, we described our novel, combined approach that i) defines the meaning of a tag ii) shares tagging data amongst users, via a use-case. Our approach allows data reuse across different tagging systems, especially given the fact that so-generated tagging data is grounded into existing Semantic Web standards and other established RDF vocabularies such as SIOC, FOAF, and DC. Thus we believe that our approach also contributes to further weaving the Semantic Web.

\section{ACKNOWLEDGMENT}

This material is based upon works supported by the Science Foundation Ireland under Grant No. SFI/02/CE1/I131. Special thanks go to Richard Cyganiak, who offered constructive criticism of this work as it progressed.

\footnotetext{
${ }^{20}$ http://wiki.foaf-project.org/SparqlPress
}

\section{REFERENCES}

[1] Scott Golder and Bernardo A. Huberman. The structure of collaborative tagging systems. Journal of Information Sciences, 32(2):198-208, April 2006.

[2] Cameron Marlow, Mor Naaman, Danah Boyd, and Marc Davis. Ht06, tagging paper, taxonomy, flickr, academic article, to read. In HYPERTEXT '06: Proceedings of the seventeenth conference on Hypertext and hypermedia, pages 31-40, New York, NY, USA, 2006. ACM Press.

[3] Ian Davis. Why tagging is expensive, September 2005. Available at: http://blogs.talis.com/panlibus/archives/2005/09/why_tagging_is.php.

[4] Tom Gruber. Collective knowledge systems: Where the social web meets the semantic web. Journal of Web Semantics, 6(1):4-13, 2008.

[5] Thomas Gruber. Ontology of folksonomy: A mashup of apples and oranges. Intl Journal on Semantic Web \& Information Systems, 3(2), 2007. Available at: http://tomgruber.org/writing/ontology-offolksonomy.htm.

[6] Peter Mika. Ontologies are us: A unified model of social networks and semantics. In International Semantic Web Conference, LNCS, pages 522-536. Springer, 2005.

[7] Richard Newman, Danny Ayers, and Seth Russell. Tag ontology, December 2005. Available at: http://www.holygoat.co.uk/owl/redwood/0.1/tags/.

[8] Hak Lae Kim, Sung Kwon Yang, John G. Breslin, and Hong Gee Kim. Simple algorithms for representing tag frequencies in the scot exporter. In IAT, pages 536-539. IEEE Computer Society, 2007.

[9] Alexandre Passant and Philippe Laublet. Meaning Of A Tag: A collaborative approach to bridge the gap between tagging and Linked Data. In Proceedings of the $W W W$ 2008 Workshop Linked Data on the Web (LDOW2008), Beijing, China, Apr 2008.

[10] Torber Knerr. Tagging ontology- towards a common ontology for folksonomies, 2006. Available at: http://tagont.googlecode.com/files/TagOntPaper.pdf.

[11] Uldis Bojars and John G. Breslin (eds.). Sioc core ontology specification, June 2007. URL http://www.w3.org/Submission/sioc-spec/.

[12] Alistair Miles and Dan Brickley. Skos core vocabulary specification, April 2005. Available at: http://www.w3.org/TR/swbp-skos-core-spec.

[13] Michel Buffa, Fabien Gandon, Guillaume Ereteo, Peter Sander, and Catherine Faron. Sweetwiki: A semantic wiki. Web Semantics: Science, Services and Agents on the World Wide Web, 6:84-97, February 2008.

[14] Tim Berners-Lee. Linked data, July 2006. Available at: http://www.w3.org/DesignIssues/LinkedData.html.

[15] Chris Bizer, Richard Cyganiak, and Tom Heath. How to publish linked data on the web, July 2007. Available at: http://www4.wiwiss.fuberlin.de/bizer/pub/LinkedDataTutorial. 